\title{
Oak Ridge Research Reactor Quarterly Report July, August, and September of 1976
}

\author{
S. S. Hurt, III \\ E. D. Lance
}




\section{DISCLAIMER}

This report was prepared as an account of work sponsored by an agency of the United States Government. Neither the United States Government nor any agency Thereof, nor any of their employees, makes any warranty, express or implied, or assumes any legal liability or responsibility for the accuracy, completeness, or usefulness of any information, apparatus, product, or process disclosed, or represents that its use would not infringe privately owned rights. Reference herein to any specific commercial product, process, or service by trade name, trademark, manufacturer, or otherwise does not necessarily constitute or imply its endorsement, recommendation, or favoring by the United States Government or any agency thereof. The views and opinions of authors expressed herein do not necessarily state or reflect those of the United States Government or any agency thereof. 


\section{DISCLAIMER}

Portions of this document may be illegible in electronic image products. Images are produced from the best available original document. 

Printed in the United States of America. Available from
National Technical Information Service

U.S. Department of Commerce 5285 Port Royal Road, Springfield, Virginia 22161

Price: Printed Copy $\$ 4.00 ;$ Microfiche $\$ 3.00$

This report was prepared as an account of work sponsored by the United States Government. Neither the United States nor the Energy Research and Development Administration/United States Nuclear Regulatory Commission, nor any of their employees, nor any of their contractors, subcontractors, or their employees, makes any warranty, express or implied, or assumes any legal liability or responsibility for the accuracy, completeness or usefulness of any information, apparatus, product or process disclosed, or represents that its use would not infringe privately owned rights. 
ORNL / TM-5832

Contract No. W-7405-eng-26

OPERATIONS DIVISION

OAK RIDGE RESEARCH REACTOR QUARTERLY REPORT

JULY, AUGUST, AND SEPTEMBER OF 1976

S. S. Hurt, III

E. D. Lance

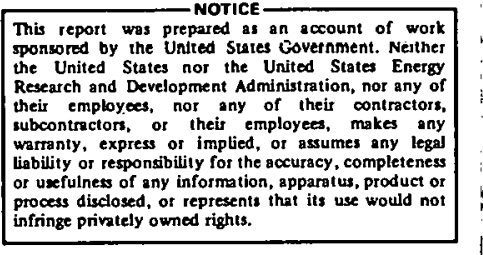

Date Published - March, 1977

NOTICE This document contains information of a preliminary nature. It is subject to revision or correction and therefore does not represent a final report.

OAK RIDGE NATIONAL LABORATORY

Oak Ridge, Tennessee 37830

operated by

UNION CARBIDE CORPORATION

for the

ENERGY RESEARCH AND DEVELOPMENT ADMINISTRATION 
THIS PAGE

\section{WAS INTENTIONALLY \\ LEFT BLANK}




\section{CONTENTS}

Summary. . . . . . . . . . . . . . . . . . . 1

Operations ......................... . . 1

Fuel Usage and Inventory. . . . . . . . . . . . . . . . . . 6

Shutdowns and Power Reductions . . . . . . . . . . . . . . . 7

Instrumentation and Reactor Controls . . . . . . . . . . . . . . 10 .

Process System and Mechanical Components. . . . . . . . . . . . 10

Experiment Facilities and Core Changes . . . . . . . . . . . . 10

In-Service Inspections . . . . . . . . . . . . . . . . 26

Distribution . . . . . . . . . . . . . . . . . 27 
SUMMARY

The ORR operated at an average power level of $29.8 \mathrm{Mw}$ for $50.9 \%$ of the time during July, August, and September of 1976. One fuel element was declared spent ( $62.4 \%$ burnup) during the quarter, while five new elements were placed in service. Two shim-rod élements were retired from service at an average burnup of $69.0 \%$.

The reactor was shut down on twelve occasions, all of which were scheduled. Reactor downtime needed for refueling, maintenance and checks was quite low, with the reactor remalning available for operation $95.5 \%$ of the time.

Maintenance activities, both mechanical and instrument were essentially routine in nature.

In-service inspections completed during the quarter included:

1. Inpsection of the decay tank strainer on 9-21-76.

2. Inspection of the reactor primary heat exchanger No. 3, secondary side, on 9-24-76.

\section{OPERATIONS}

The basic operating data presented in Table 1 indicates that the ORR operation for the quarter was normal as compared with recent experience.

Details relative to cycles of operation during the quarter are given in Table 2, while Figures 1, 2, and 3 are plots of shim-rod position versus time for these cycles. 
Table 1. ORR Basic. Operating Data

Period: July, August and September, 1976

\begin{tabular}{|c|c|c|c|c|}
\hline & $\begin{array}{c}\text { This } \\
\text { Quarter }\end{array}$ & $\begin{array}{l}\text { Last } \\
\text { Quarter. }\end{array}$ & $\begin{array}{l}\text { Year } \\
\text { To Date }\end{array}$ & $\begin{array}{l}\text { January Through } \\
\text { September, } 1975\end{array}$ \\
\hline Total energy, Mwd & $\cdot 1,394: 7$ & $2,434.8$ & $6,083.9$ & $6,949.1$ \\
\hline $\begin{array}{l}\text { Average power, } \\
\text { Mw/operating } \mathrm{hr} .\end{array}$ & 29.8 & 29.7 & 29.8 & 29.8 \\
\hline Time operating, \% & $50.9 *$ & 90.0 & 74.6 & 85.9 \\
\hline Availability, \% & 95.5 & 95.5 & 93.9 & 89.6 \\
\hline $\begin{array}{l}\text { Reactor water radio- } \\
\text { activity } \frac{\mathrm{d} / \mathrm{min}}{\mathrm{ml}} \text { (av) }\end{array}$ & 28,300 & 29,400 & 28,600 & 30,400 \\
\hline $\begin{array}{l}\text { Pool water radio- } \\
\text { activity } \frac{\mathrm{c} / \mathrm{min}}{\mathrm{m} 1}(\mathrm{av})\end{array}$ & 590 & 580 & 593 & 650 \\
\hline $\begin{array}{l}\text { Reactor water resis- } \\
\text { tivity, ohm-cm (av) }\end{array}$ & $1,234,000$ & 844,000 & 967,000 & 854,000 \\
\hline $\begin{array}{l}\text { Pool water resis- } \\
\text { tivity, ohm-cm (av) }\end{array}$ & $.784,000$ & 700,000 & 761,000 & 791,000 \\
\hline Fuel elements depleted & 1 & 9 & 12 & 73 \\
\hline $\begin{array}{l}\text { Average burnup of fuel } \\
\text { elements depleted, } \%\end{array}$ & 62.4 & 55.3 & 56.5 & 52.3 \\
\hline Shim rods depleted & 2 & 2 & 8 & 10 \\
\hline $\begin{array}{l}\text { Average burnup of shim } \\
\text { rods depleted, } \%\end{array}$ & 69.0 & 62.5 & 65.1 & 64.8 \\
\hline Radioisotope samples & 2 & 2 & 4 & 25 \\
\hline Research samples & 0 & 5 & 6 & .41 \\
\hline
\end{tabular}

Table 2. Cycles of Operation

\begin{tabular}{clcc}
\hline Cycle No. & Date Beguñ & Date Ended & $\begin{array}{c}\text { Accumulated Energy } \\
\text { (Mwd) }\end{array}$ \\
\hline 1.32 & May 27, 1976 & Aligust 2, 1976 & $* * 1891.9$ \\
133 & August 10,197.6 & September 12, 1976 & 406.8 \\
134 & September 27, 1976 & In progress. . & 107.1 \\
\hline
\end{tabular}

* Time operating (\%) low due to reduced request for operation. $* *$

MWD this quarter -880.8 


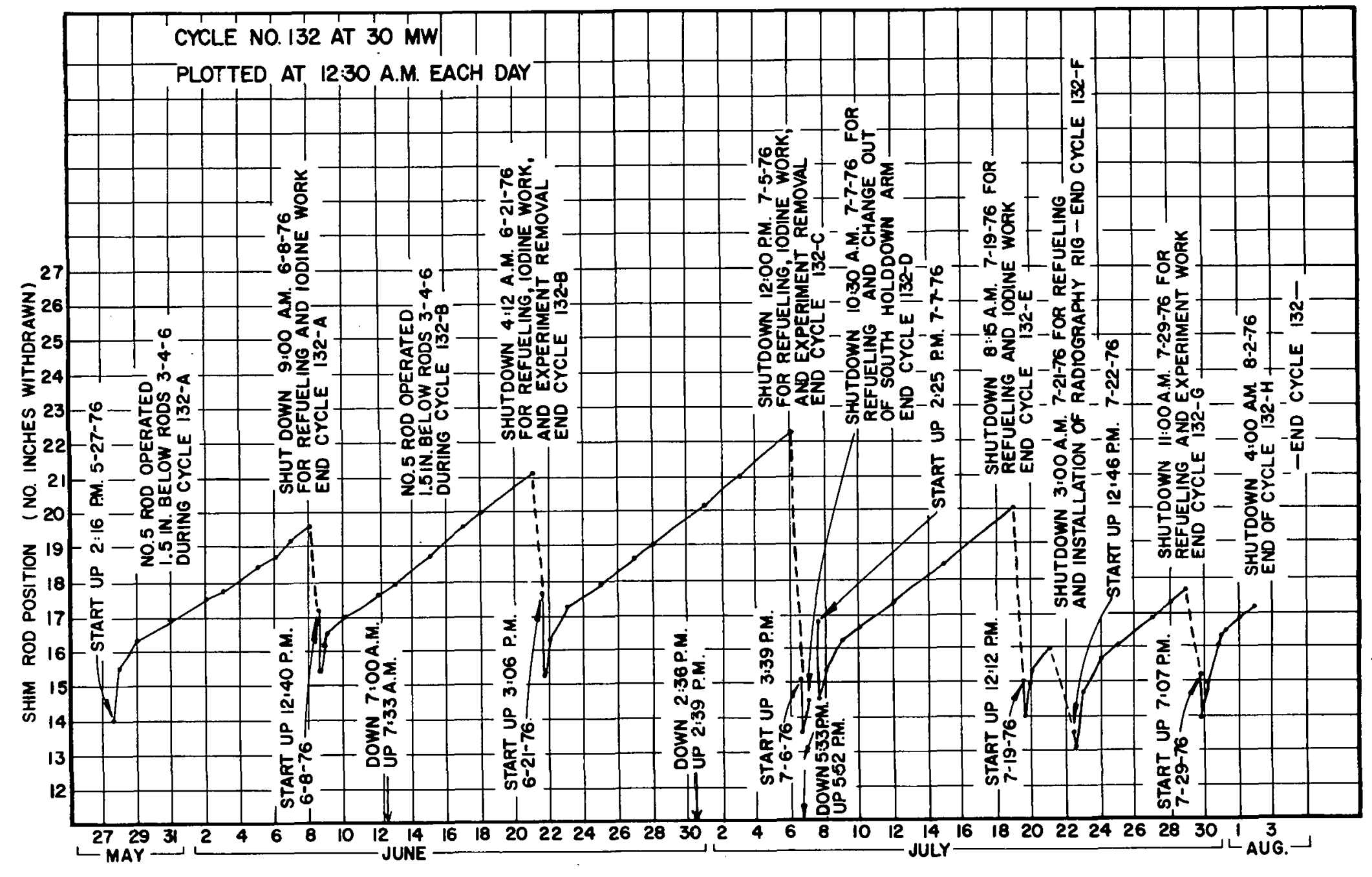

Fig. 1. Shim Rod Position vs. Time (Cycle 132) 


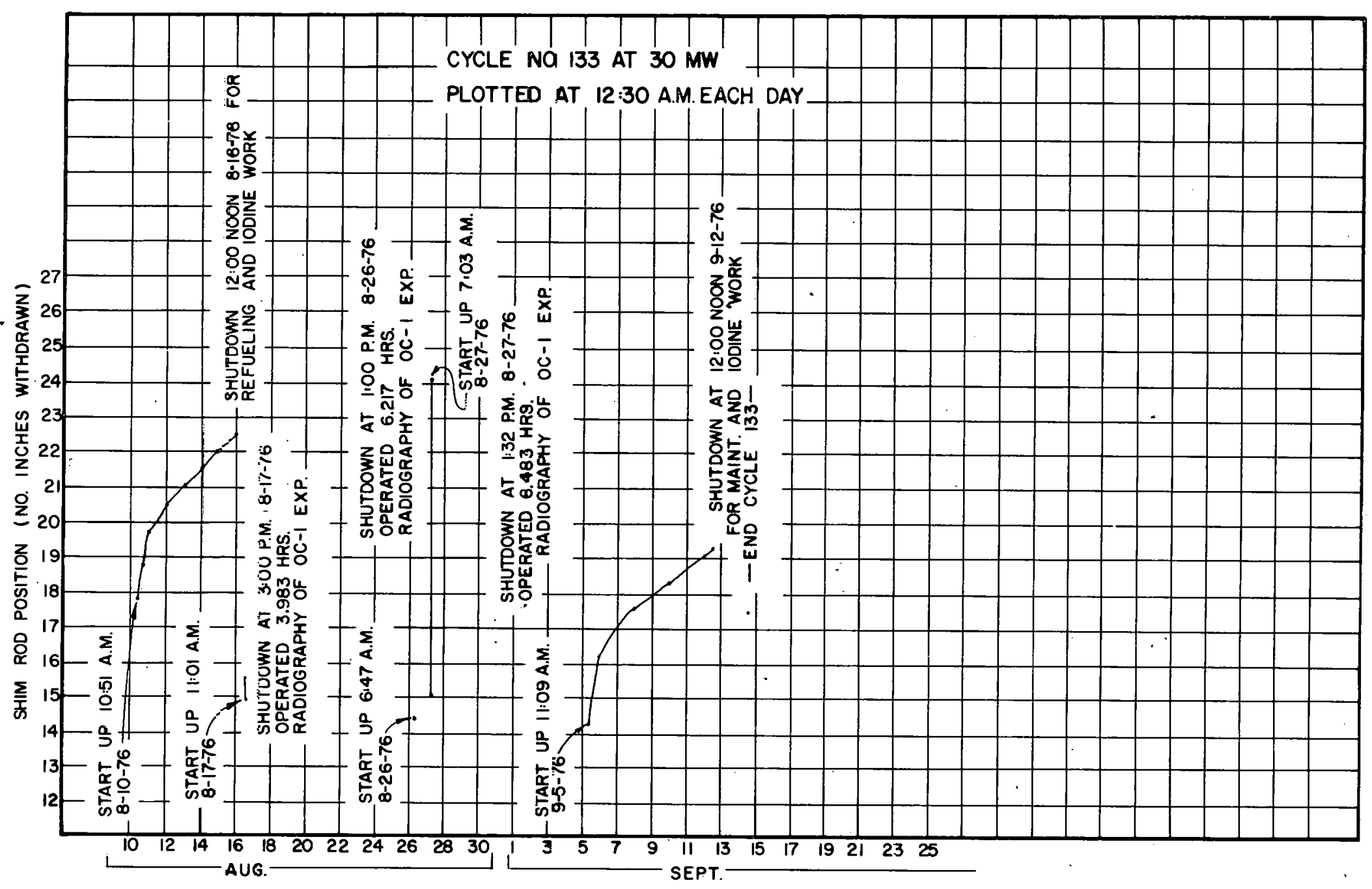

Fig. 2. Shim Rod Position vs. Time (Cycle 1ミ3) 


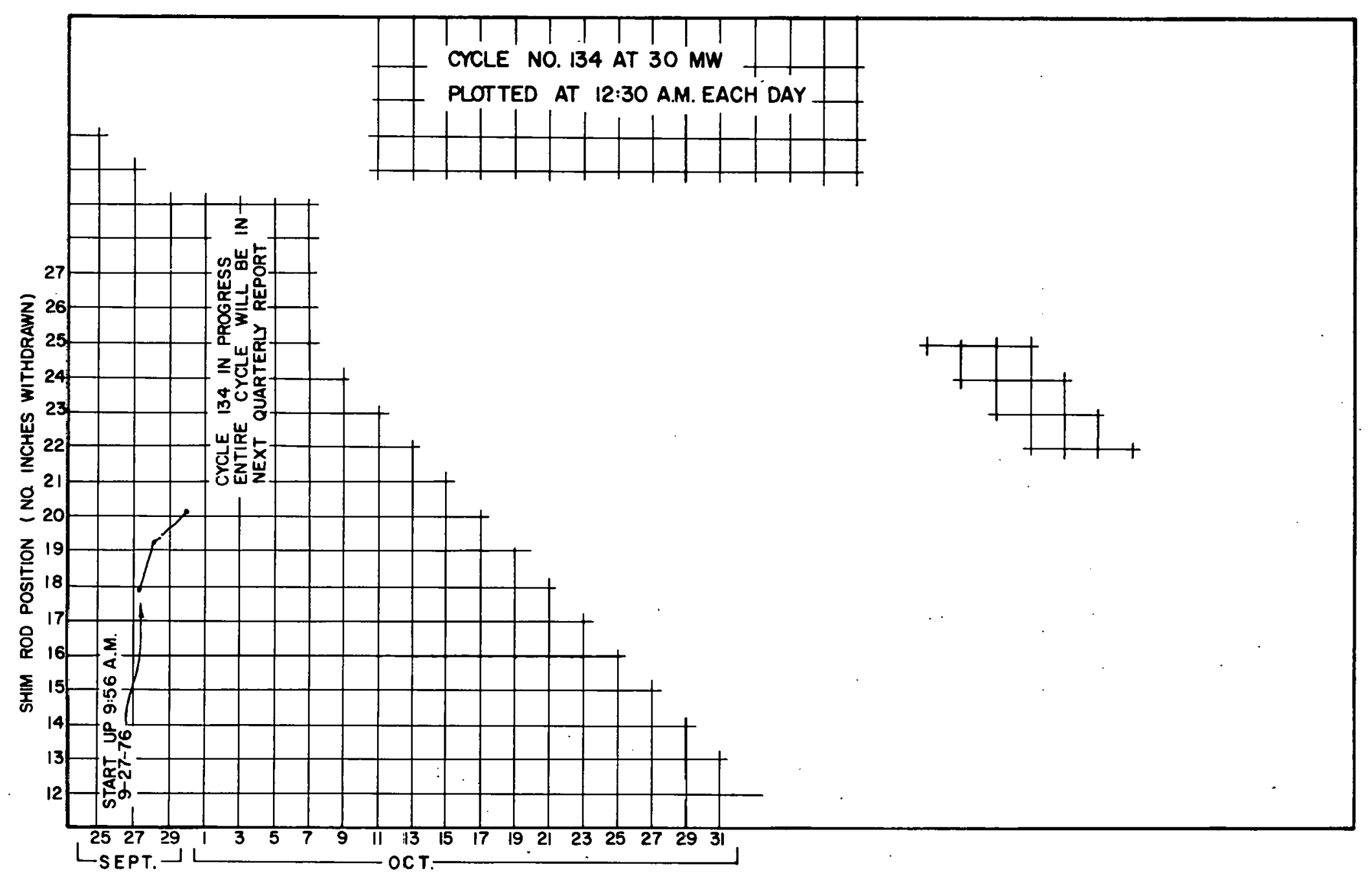

Fig. 3. Shim Rod Pósition vs. Time (Cycle 134) 
FUEL USAGE AND INVENTORY

Due to curtalled operation only five new fuel elements and two new shim rods were placed in service during the quarter.

other details of fuel usage and inventory may be found.in. Tables

1 and 3 .

Table.3. ORR Fuel. Status

\begin{tabular}{|c|c|c|c|c|}
\hline & $\begin{array}{l}\text { This } \\
\text { Quarter }\end{array}$ & $\begin{array}{l}\text { Last: } \\
\text { Quarter }\end{array}$ & $\begin{array}{c}\text { Jan-Sept } \\
1976\end{array}$ & $\begin{array}{c}\text { Jan-Sept } \\
1975\end{array}$ \\
\hline $\begin{array}{l}\text { Depleted fuel elements trans- } \\
\text { ferred for chemical separa- } \\
\text { tion. }\end{array}$ & 13 & 0 & 58 & 121 \\
\hline $\begin{array}{l}\text { Average percent burnup of fuel } \\
\text { element transferred }\end{array}$ & 53.8 & - & 52.3 & 48.9 \\
\hline $\begin{array}{l}\text { New fuel elements placed in } \\
\text { service }\end{array}$ & 5 & 25 & 58 & 75 \\
\hline $\begin{array}{l}\text { New fuel-elements available } \\
\text { for use }\end{array}$ & $* 63$ & 68 & & \\
\hline $\begin{array}{l}\text { Depleted shim rods transferred } \\
\text { for chemical separation }\end{array}$ & 4 & 0 & 10 & 18 \\
\hline $\begin{array}{l}\text { Average percent burnup of shim } \\
\text { rods transferred. }\end{array}$ & 6.3 .0 & - & 64.1 & $64: 5$ \\
\hline New shim rods placed in service & 2 & 2 & 8 & 10 \\
\hline New shim rods available for use & 2 & 0 & & \\
\hline
\end{tabular}


SHUTDOWNS AND POWER REDUCTIONS

Reactor downtime (power level $<\mathrm{N}_{\mathrm{L}}$ ) totaled approximately 1,085 hours. A summary of the shutdowns is given in Table. 4, and details of each are contained in Table 5. Table 6 offers information relative to power reductions which did not result in a shutdown.

Table 4. Summary of Shutdowns

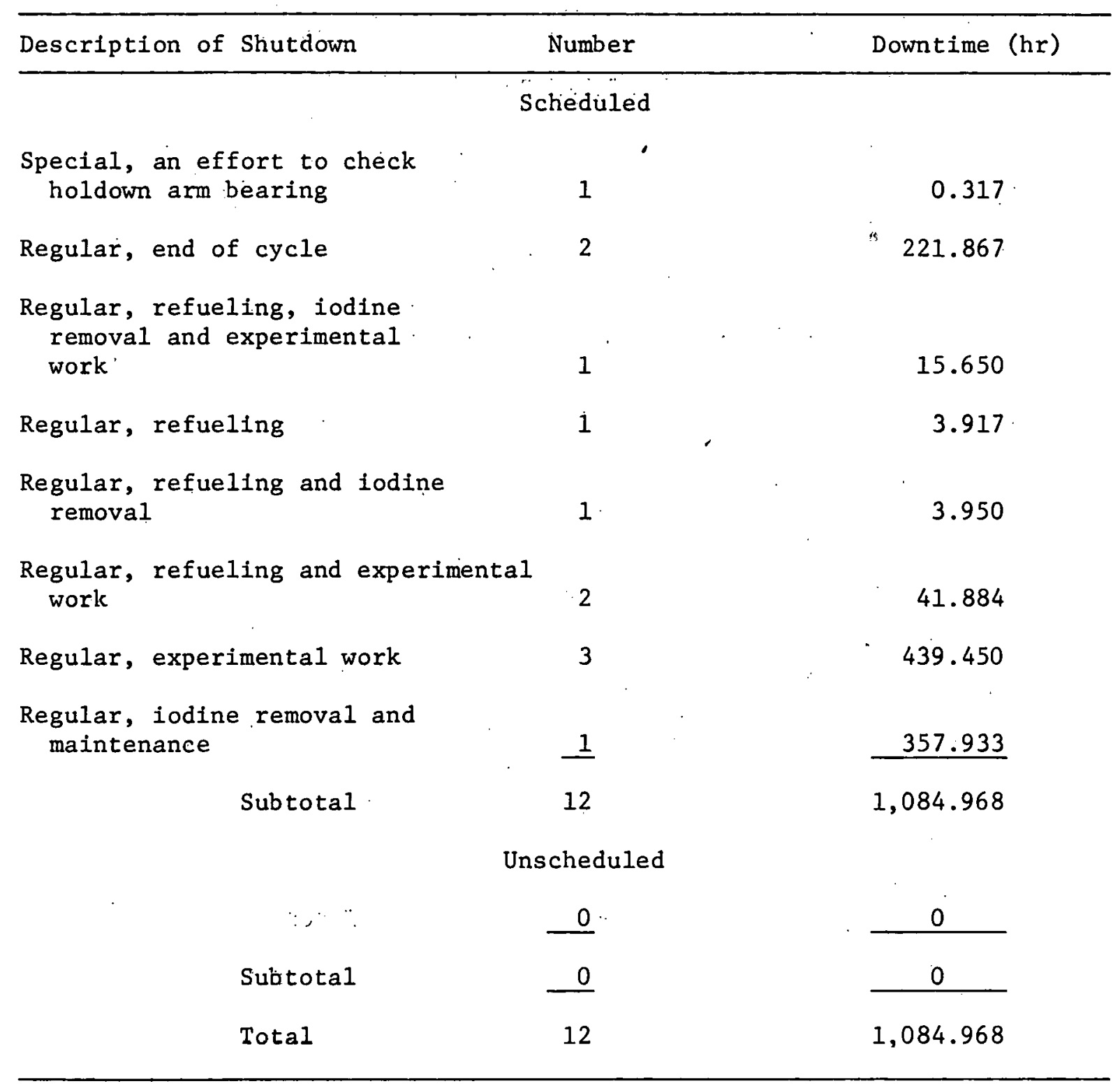


Table 5. ORR Scheduled Shutdowns, Details

\begin{tabular}{|c|c|c|c|}
\hline Date. & $\begin{array}{l}\text { Duration } \\
\text { (hr) }\end{array}$ & $\begin{array}{c}\text { Enci } \\
\text { Cycle }\end{array}$ & $R \in$ narks \\
\hline $7-6-76$ & 15.650 & $\begin{array}{ll}132 C \\
.\end{array}$ & $\begin{array}{l}\text { The reactor was refueled and isotope (iodine, xenor.) work was completed. } \\
\text { Experimert work consisted of removing an experiment from core position E-3. }\end{array}$ \\
\hline $7-6-76$ & $0 . .317$ & - & $\begin{array}{l}\text { The reactor was scrammed in an effort to check the possibility of a stuck } \\
\text { bearing in the south hold down arm. }\end{array}$ \\
\hline $7-7-76$ & 3.917 & $132 \mathrm{D}$ & The reactor was refueled. The south hold down arm was replaced. \\
\hline $7-19-76$ & 3.950 & $132 \mathrm{E}$ & The reactor was refueled and isotope (iodize, xenon) work was completed. \\
\hline $7-21-76$ & 33.767 & $132 \vec{F}$ & $\begin{array}{l}\text { The reactor was refueled. Experiment work consisted of the installation of } \\
\text { the radiography facility in the reactor } 2001 \text { and removal of experiment } \\
\text { GB-10 from the poolside facility for ne:ltron radiography. }\end{array}$ \\
\hline $7-29-76$ & 8.117 & $132 \mathrm{G}$ & $\begin{array}{l}\text { The reactor was refueled. Experiment work consisted of the reinstallation of } \\
\text { the GB-10 experiment in the poolside facility. }\end{array}$ \\
\hline $8-2-76$ & 198.850 & $132 \mathrm{E}$ & 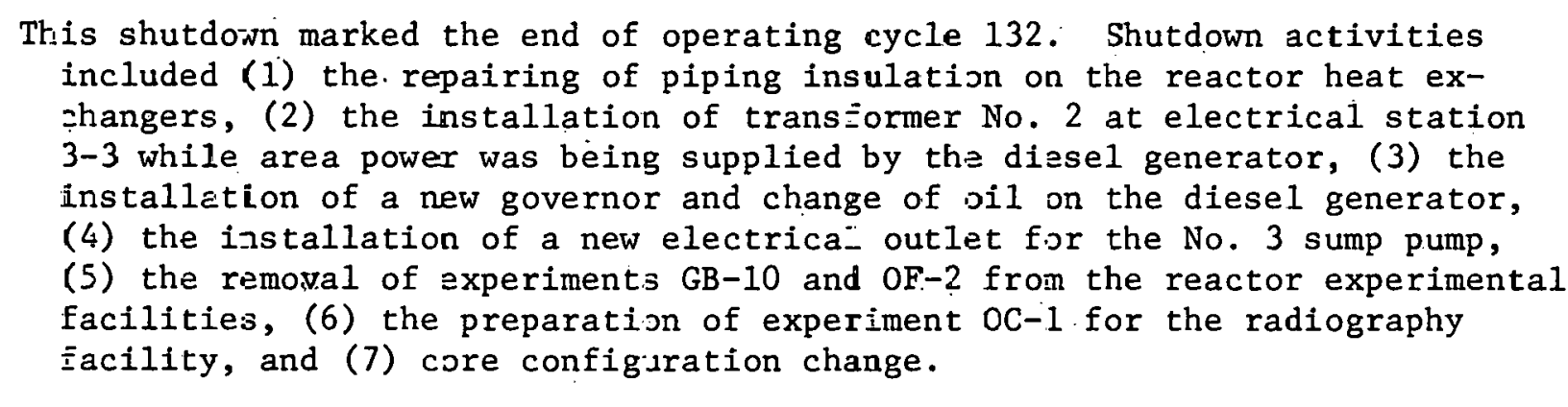 \\
\hline $8-16-76$ & 23.017 & $133 A$ & $\begin{array}{l}\text { The shutdown activities included (1) refueling, (2) iodine-xenon work, and } \\
\text { (3) programmed maintenance. }\end{array}$ \\
\hline
\end{tabular}


Table 5 (continued)

\begin{tabular}{|c|c|c|c|}
\hline Date & $\begin{array}{l}\text { Duration } \\
\text { (hr) }\end{array}$ & $\begin{array}{l}\text { End } \\
\text { Cycle }\end{array}$ & Remarks \\
\hline $8-17-76$ & 207.783 & - & $\begin{array}{l}\text { The reactor was shut down after unsuccessful attempts to obtain } \\
\text { radiographs of experiment } 0 C-1 \text { in the neutron radiography } \\
\text { facility. }\end{array}$ \\
\hline $8-26-76$ & 18.050 & - & $\begin{array}{l}\text { The reactor was shut down after two radiographs were made of the } \\
\text { oc-1 experiment in the neutron radiography facility. The results } \\
\text { were not satisfactory due to the type of film used. }\end{array}$ \\
\hline $8-27-76$ & 213.617 & - & $\begin{array}{l}\text { This shutdown ended a successful attempt to obtain radiographs of } \\
\text { the oC-1 experiment in the neutron radiography facility. }\end{array}$ \\
\hline $9-12-76$ & 357.933 & $133 B$ & $\begin{array}{l}\text { The reactor was shut down after radiographing of the OF-2 exper- } \\
\text { iment in the neutron radiography facility. Isotope (iodine- } \\
\text { xenon) work was completed. The routine end-of-operating-cycle } \\
\text { maintenance was started on } 9-12-76 \text { and completed on } 9-22-76 \text {. }\end{array}$ \\
\hline
\end{tabular}

Table 6. Reductions in ORR Power Not Resulting in Shutdowns

\begin{tabular}{|c|c|c|c|c|}
\hline Date & $\begin{array}{l}\text { Source of } \\
\text { Signal }\end{array}$ & $\begin{array}{l}\text { Type of } \\
\text { Signal }\end{array}$ & $\begin{array}{l}\text { Lowest } \\
\text { Power. (Mw) }\end{array}$ & Comments \\
\hline $8-26-76$ & Setback & Automatic & $\mathrm{N}_{\mathrm{L}}$ & $\begin{array}{l}\text { The reactor primary water by-pass valve } \\
\text { closed due to demand for cooler water. The } \\
\Delta \mathrm{P} \text { decreased to the } 22: 9 \text { PSI setpoint. }\end{array}$ \\
\hline $9-9-76$ & Setback & Automatic & 6 & $\begin{array}{l}\text { The reactor primary water by-pass valve } \\
\text { closed when switching from manual to auto- } \\
\text { matic position. The } \Delta \mathrm{P} \text { decreased to the } \\
22.9 \mathrm{PSI} \text { setpoint. }\end{array}$ \\
\hline
\end{tabular}




\section{INSTRUMENTATION AND REACTOR CONTROLS}

The performance of the instrumentation for the complex was quite satisfactory and only rather routine maintenance was required as indicated in Table 7. Table 8 reflects the status of the various fission and ionization chambers.

PROCESS SYSTEM AND MECHANICAL COMPONENTS

Details of maintenance or changes to the process system are given in Table 9, while Table 10 lists the maintenance on the mechanical components. Table 11 summarizes the results of efficiency tests of the various gaseous waste filters.

\section{EXPERIMENT FACILITIES AND CORE CHANGES}

Experiment facility usage is given in Table 12. The core configurations used during the quarter are shown in Figure Nos. 4 and 5 .

PROCEDURES, ADDITIONS, AND/OR REVISIONS

There were two revisions to the "ORR Operating Manual" listed in Table 13. 
For Fuel Cycles 132-C, D, E, F, G, H

\begin{tabular}{|c|c|c|c|c|c|c|c|c|}
\hline 1 & 2 & 3 & 4 & 5 & 6 & 7 & 8 & 9 \\
\hline $\mathrm{Be}$ & $\mathrm{Be}$ & $\mathrm{Be}$ & $F$ & $F$ & $F$ & $\mathbf{F}$ & $F$ & $\mathrm{Be}$ \\
\hline $\mathrm{Be}$ & $\mathrm{Be}$ & F & SR & $F$ & SR & $F$ & ${ }^{131_{I}}$ & $\mathrm{Be}$ \\
\hline $\mathrm{Be}$ & $\mathrm{Be}$ & $F$ & $F$ & $F$ & $\mathrm{~F}$ & $F$ & $F$ & $\mathrm{Be}$ \\
\hline $\mathrm{Be}$ & $\mathrm{F}$ & $F$ & SR & F & SR & $\mathbf{F}$ & HT & $\mathrm{Be}$ \\
\hline $\mathrm{Be}$ & F & F & $F$ & $F$ & $F$ & E & F & $\mathrm{Be}$ \\
\hline $\mathrm{Be}$ & $\mathrm{Be}$ & F & SR & F & SR & F & $F$ & $\mathrm{Be}$ \\
\hline $\mathrm{Be}$ & $\mathrm{Be}$ & $\mathrm{Be}$ & $\mathrm{Be}$ & $\mathrm{Be}$ & $\mathrm{Be}$ & $\mathrm{Be}$ & $\mathrm{Be}$ & $\mathrm{Be}$ \\
\hline
\end{tabular}

LATTICE COMPONENT

NUMBER

Fuel (F)

$\underline{28}$

Shim Rod (SR)

6

Beryllium (Be)

26

Experiment (E)

1

Iodine Slecve $\left({ }^{131} I\right)$

1

Isotope Stringer (Iso)

Hydraulic Tube (HT)

Fig. 4. ORR Lattice Configuration-July 1, 1976, through August 4, 1976. 
For Fuel Cycles 133A, B, 134A

\begin{tabular}{|c|c|c|c|c|c|c|c|c|}
\hline 1 & 2 & 3 & 4 & 5 & 6 & 7 & 8 & 9 \\
\hline $\mathrm{Be}$ & $\mathrm{Be}$ & $\mathrm{Be}$ & $\mathrm{F}$ & $\mathrm{F}$ & $\mathrm{F}$ & $\mathrm{Be}$ & $\mathrm{Be}$ & $\mathrm{Be}$ \\
\hline $\mathrm{Bt}^{\prime}$ & $B_{t}$ & F & SR & $F$ & SR & $F$ & $\mathrm{Be}$ & $\mathrm{Be}$ \\
\hline $\mathrm{Be}$ & $\mathrm{Be}$ & $F$ & $\mathrm{~F}$. & $F$ & $F$ & $F$ & $F$ & $\mathrm{Be}$ \\
\hline $\mathrm{Be}$ & $\mathrm{Be}$ & $F$ & SR & $F$ & SR & $\mathrm{F}$ & $\mathrm{HT}$ & $\mathrm{Be}$ \\
\hline $\mathrm{Be}$ & $\mathrm{Be}$ & $F$ & F & $\mathrm{F}$ & $F$ & $131_{I}$ & $F$ & $\mathrm{Be}$ \\
\hline $\mathrm{Be}$ & $\mathrm{Be}$ & $\mathrm{F}$ & SR & F & SR & $F$ & $F$ & $\mathrm{Be}$ \\
\hline $\mathrm{Be}$ & $\mathrm{Be}$ & $\mathrm{Be}$ & $\mathrm{Be}$ & $\mathrm{Be}$ & $\mathrm{Be}$ & $\mathrm{Be}$ & $\mathrm{Be}$ & $\mathrm{Be}$ \\
\hline
\end{tabular}

LATTICE COMPONENT

Fue1 ( $F)$

Shim Rod (SR)

Beryl11un (Be)

Lxperimene (E)

Iodine sleeve $\left({ }^{131} I\right)$

Isotope Stringer (Iso)

Hydraulic Tube (HT)
NUMBER
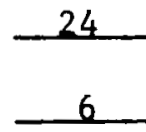

31
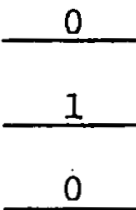

1

Fig. 5. ORR Lattice Configuration-August 4, 1976, through September 30, 1976. 
Table 7. Maintenance and Changes, Instrumentation and Controls

\begin{tabular}{llll}
\hline Date & Component & Trouble or Change & Reason or Maintenance \\
\hline
\end{tabular}

\section{No. 1 Counting-Rate Channe1}

No maintenance or changes were required.

No. 2 Counting-Rate Channel

$9-20-76$

$8-16-76$

8-10-76

\author{
No. 2 fission Chamber failure \\ chamber
}

No. 1 Log-N Channel
The unit was replaced to correct an erratic readout on the $\log -\mathrm{N}$ recorder.

The faulty chamber was replaced with chamber TSM-4-566.

L.og-N recorder Replacement.
power supply.
No. 2 Log-N Channel

Log-N amplifier Replacement
power supply

The faulty power supply was replaced.

No. 1 Safety Channel

No maintenance or changes were required.

No. 2 Safety Channe1

No maintenance or changes were required.

No. 3 Safety Channe1

No maintenance or changes were required. 
Table 7 (continued)

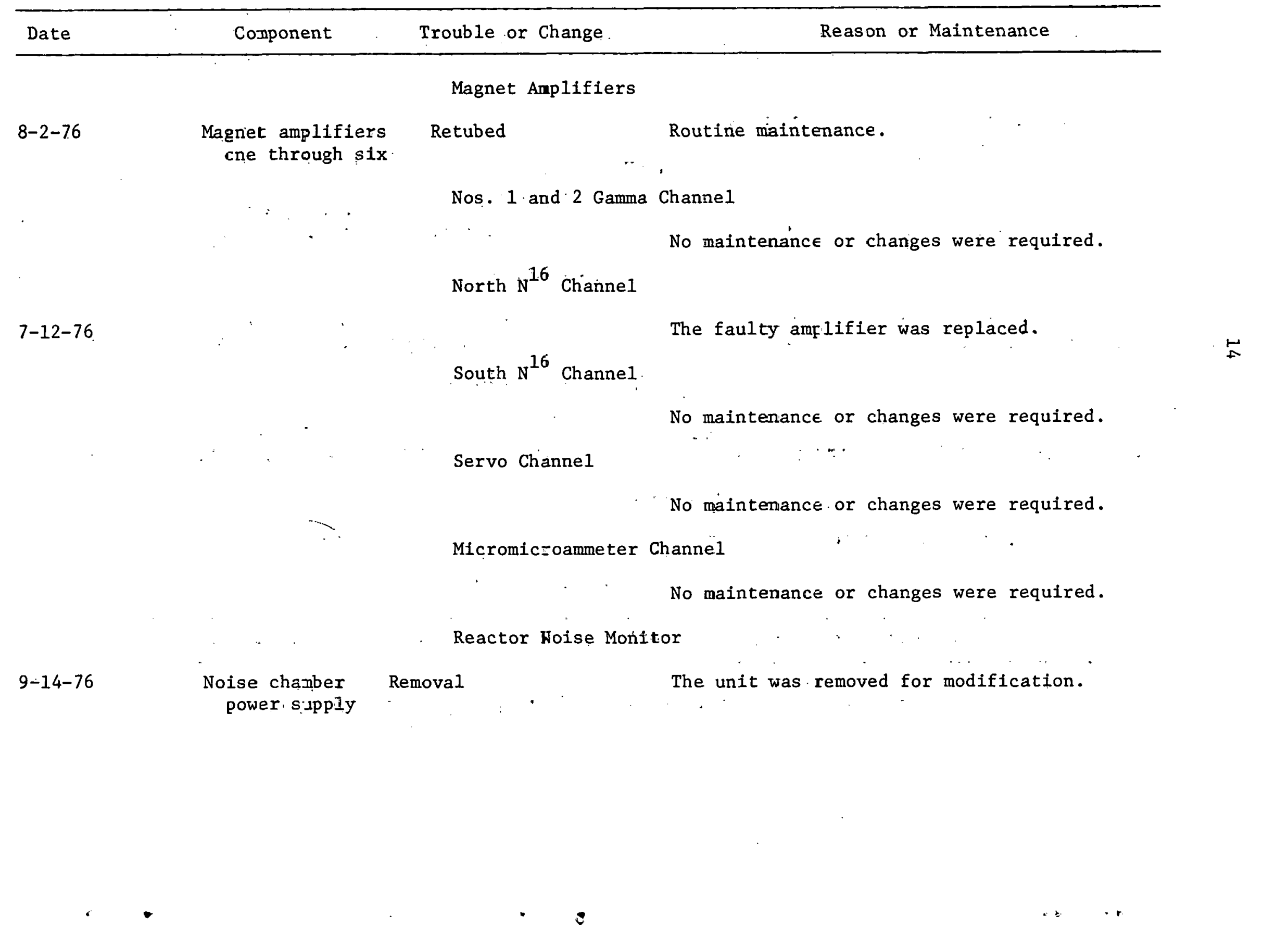


Table 7 (continued)

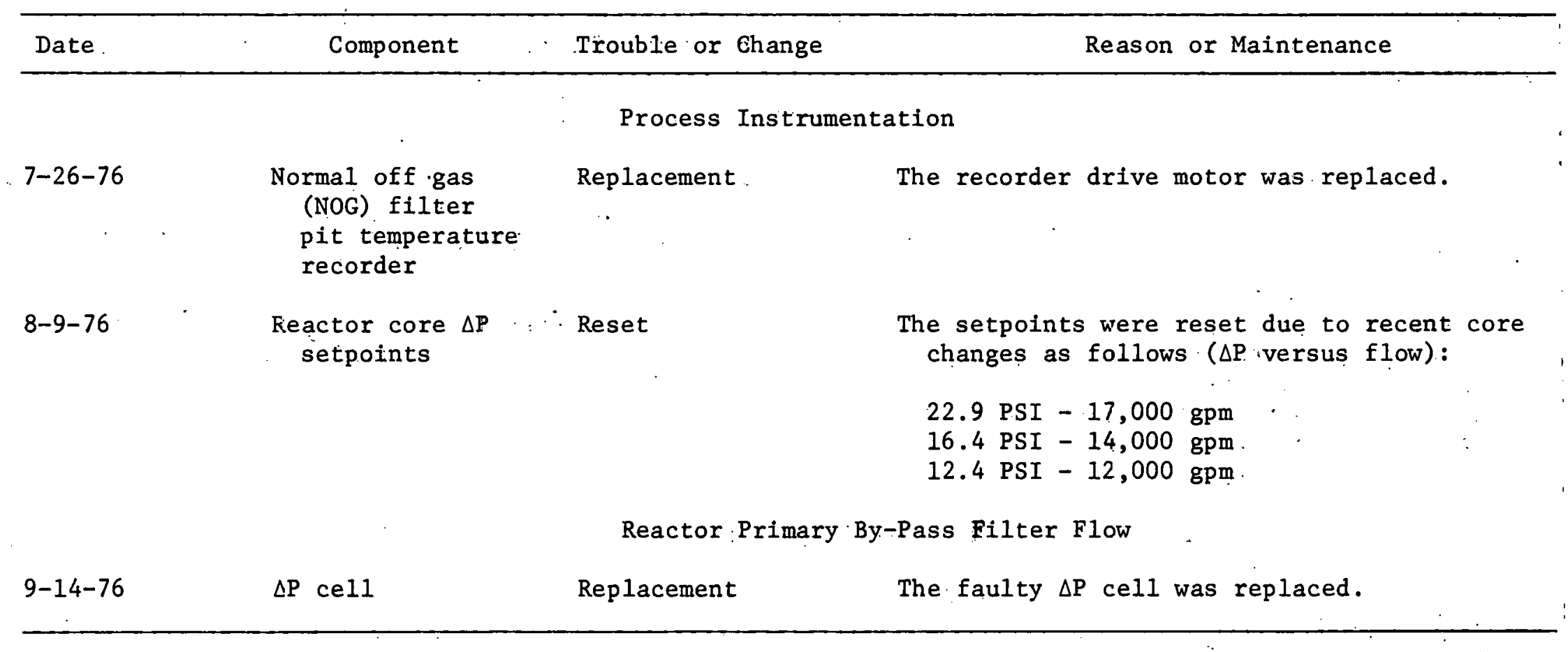


Table 8. Present Status of Ionization Chambers and Fission Chambers

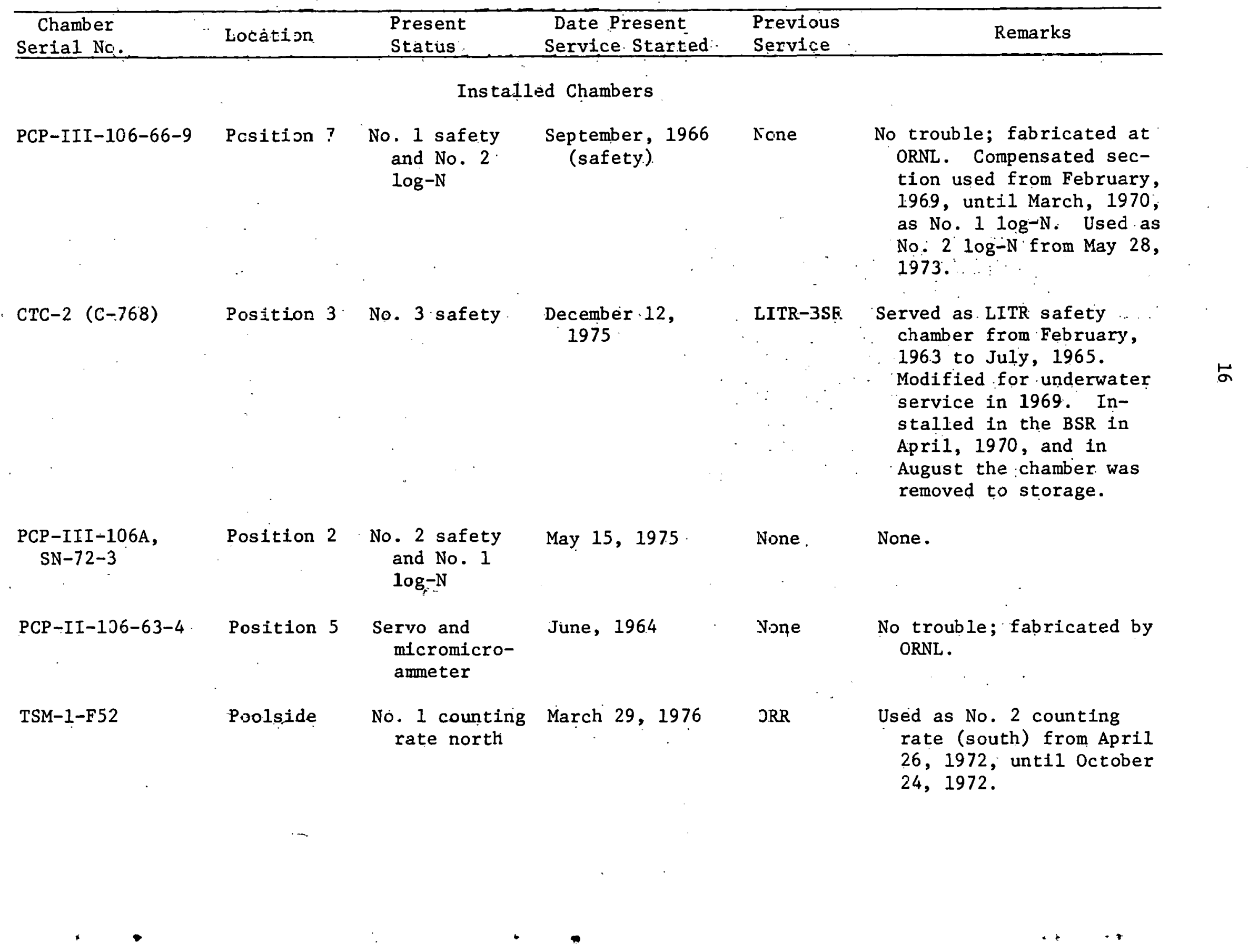


Table 8 (continued)

\begin{tabular}{|c|c|c|c|c|c|}
\hline $\begin{array}{c}\text { Chamber } \\
\text { Serial No. }\end{array}$ & Location & $\begin{array}{l}\text { Present } \\
\text { Status. }\end{array}$ & $\begin{array}{l}\text { Date Present } \\
\text { Service Started... }\end{array}$ & $\begin{array}{l}\text { Previous } \\
\text { Service }\end{array}$ & Remarks \\
\hline CTC-5 (C-770) & Position 8 & $\begin{array}{l}\text { Chamber dis- } \\
\text { connected }\end{array}$ & October; 1971 & LITR & $\begin{array}{l}\text { Used as No. } 1 \text { safety at } \\
\text { the LITR; modified for } \\
\text { ORR-BSR underwater ser- } \\
\text { vice. }\end{array}$ \\
\hline$P C P-I I-105-63-2$ & $\begin{array}{l}\text { Poolside, } \\
\text { south }\end{array}$ & $\begin{array}{l}\text { No. } 1 \text { gamma, } \\
\text { south }\end{array}$ & April 26, 1972 & ORR & $\begin{array}{l}\text { Used as No. } 2 \text { gamma, north, } \\
\text { from April, 1964, until } \\
\text { December, 1970. }\end{array}$ \\
\hline PCP-II-105-63-3 & $\begin{array}{l}\text { Poolside, } \\
\text { north }\end{array}$ & $\begin{array}{l}\text { No. } 2 \text { gamma, } \\
\text { north }\end{array}$ & August 17, 1971 & ORR & $\begin{array}{l}\text { Used as No. } 1 \text { gamma, } \\
\text { south, from Apri1, 1964, } \\
\text { until March, 1970. }\end{array}$ \\
\hline $\begin{array}{l}\text { TSM-4-566 } \\
\text { IC-2634 }\end{array}$ & Poolside & $\begin{array}{l}\text { No. } 2 \text { count:- } \\
\text { ing rate }\end{array}$ & September 20, 1976 & None. & $\begin{array}{l}\text { Replaced fission chamber } \\
\text { "snake" assembly TSM-3- } \\
\text { F29: }\end{array}$ \\
\hline & & Spare & Chambers & & \\
\hline PCP-II-105-63-1 & $\begin{array}{l}\text { Instrumenta- } \\
\text { tion \& cor- } \\
\text { trols shof }\end{array}$ & $\begin{array}{l}\text { Awaiting } \\
-\quad \text { repair }\end{array}$ & April 26, 1972 & ORR & $\begin{array}{l}\text { Used as No. } 1 \text { gamma, } \\
\text { south, from March } 31 \text {, } \\
\text { 1970, until Apri1 26, } \\
\text { 1972. }\end{array}$ \\
\hline $\mathrm{S} 27-34 \mathrm{R}-\mathrm{F} 29$ & $\begin{array}{l}\text { ORR plug } \\
\text { storage }\end{array}$ & $\begin{array}{l}\text { Awaiting } \\
\text { repair }\end{array}$ & April $26 ; 1971$ & ORR & $\begin{array}{l}\text { Used as No. } 1 \text { counting } \\
\text { rate from July } 28,1970 \text {, } \\
\text { to April } 26,1971 .\end{array}$ \\
\hline
\end{tabular}


Table 3 (continued)

\begin{tabular}{|c|c|c|c|c|c|c|}
\hline $\begin{array}{c}\text { Chamber } \\
\text { Serial No. }\end{array}$ & Locati-on & $\begin{array}{l}\text { Present } \\
\text { Status }\end{array}$ & & $\begin{array}{l}\text { Date Presert } \\
\text { Service. Started }\end{array}$ & $\begin{array}{l}\text { Previous } \\
\text { Service }\end{array}$ & Remarks \\
\hline S3-8R-F22 & $\begin{array}{l}\text { ORR pli1g } \\
\text { storage }\end{array}$ & $\begin{array}{l}\text { Awaiting } \\
\text { repair }\end{array}$ & & April 26, 1971 & aRR & $\begin{array}{l}\text { Used as No. } 2 \text { counting } \\
\text { rate from January } 14, \\
1970 \text {, to April 26, } 1971 .\end{array}$ \\
\hline CTC-1 & $\begin{array}{l}\text { Instrumenta- } \\
\text { tion \& } \\
\text { controls } \\
\text { shop }\end{array}$ & $\begin{array}{l}\text { Awaiting } \\
\text { repair }\end{array}$ & & October, $=97.1$ & QRR & $\begin{array}{l}\text { Used as noise monitor } \\
\text { from March; 1962, until } \\
\text { October 1, 1971. }\end{array}$ \\
\hline CTC-4 $(C-769)$ & $\begin{array}{l}\text { ORR plug } \\
\text { storage }\end{array}$ & & & $\infty$ & LITR & $\begin{array}{l}\text { Used as No. } 2 \text { safety in } \\
\text { the LITR; modified for } \\
\text { ORR-BSR underwater ser- } \\
\text { vice. }\end{array}$ \\
\hline PCP-III-106-66-5 & $\begin{array}{l}\text { Instrumenta- } \\
\text { tton an: } \\
\text { cont }=01 . \\
\text { shop }\end{array}$ & $\begin{array}{l}\text { Awalting } \\
\text { repair }\end{array}$ & & $\therefore \quad \therefore \quad \because \quad \because \cdot$ & ORR & $\begin{array}{l}\text { Used as No. } 2 \text { safety and } \\
\text { log }-\mathrm{N} \text { from September } 27, \\
1968 \text {, to July } 22,1970, \\
\text { and from May } 7,1971, \\
\text { to May } 20 ; 1971 \text {; from } \\
\text { November, } 1971 \text {, to } \\
\text { January } 24,1973 \text {, as } \\
\text { No. } 2 \text { safety and until } \\
\text { May } 28,1973 \text {, as No. } 2 \\
\text { log-N. }\end{array}$ \\
\hline TSM-2-F45 & I\&C shop & $\begin{array}{l}\text { Awaiting } \\
\text { repair. }\end{array}$ & 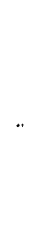 & August 8,1975 & ORR & $\begin{array}{l}\text { Used as No. } 1 \text { counting } \\
\text { rate from April } 26, \\
1971 \text {, unt1l December } 5 \text {, } \\
1972 \text {. . Removed } 3 / 30 / 76 \\
\text { to shop for repair. }\end{array}$ \\
\hline
\end{tabular}


Table 8 (continued)

\begin{tabular}{|c|c|c|c|c|c|}
\hline $\begin{array}{l}\text { Chamber } \\
\text { Serial No. }\end{array}$ & Location & $\begin{array}{l}\text { Present } \\
\text { Status }\end{array}$ & $\begin{array}{l}\text { Date Present } \\
\text { Service Started }\end{array}$ & $\begin{array}{l}\text { Present } \\
\text { Service }\end{array}$ & Remarks \\
\hline$P C P-I I-106-66-3$ & I\&C shop & $\begin{array}{l}\text { Awaiting } \\
\text { repair }\end{array}$ & $\begin{array}{l}\text { May, } 1974 \text { (safety) } \\
\text { March, } 1970 \\
(10 g-N) \text { removed } \\
\text { from service } \\
\text { December } 12,1975\end{array}$ & $\begin{array}{l}\text { None } \\
5\end{array}$ & $\begin{array}{l}\text { Fabricated by ORNL. } \\
\text { Chamber failed December } \\
12,1975 \text {. Removed } \\
9 / 2 / 76 \text { to shop for } \\
\text { repair. }\end{array}$ \\
\hline TSM-3-F29 & I\&C shop & $\begin{array}{l}\text { Awaiting } \\
\text {-repair }\end{array}$ & . & ORR & $\begin{array}{l}\text { Used as No. } 2 \text { counting } \\
\text { rate from October } 24 \text {, } \\
1972 \text {, until September } \\
29,1976 .\end{array}$ \\
\hline
\end{tabular}


Table 9. Process Systems, Maintenance and Changes

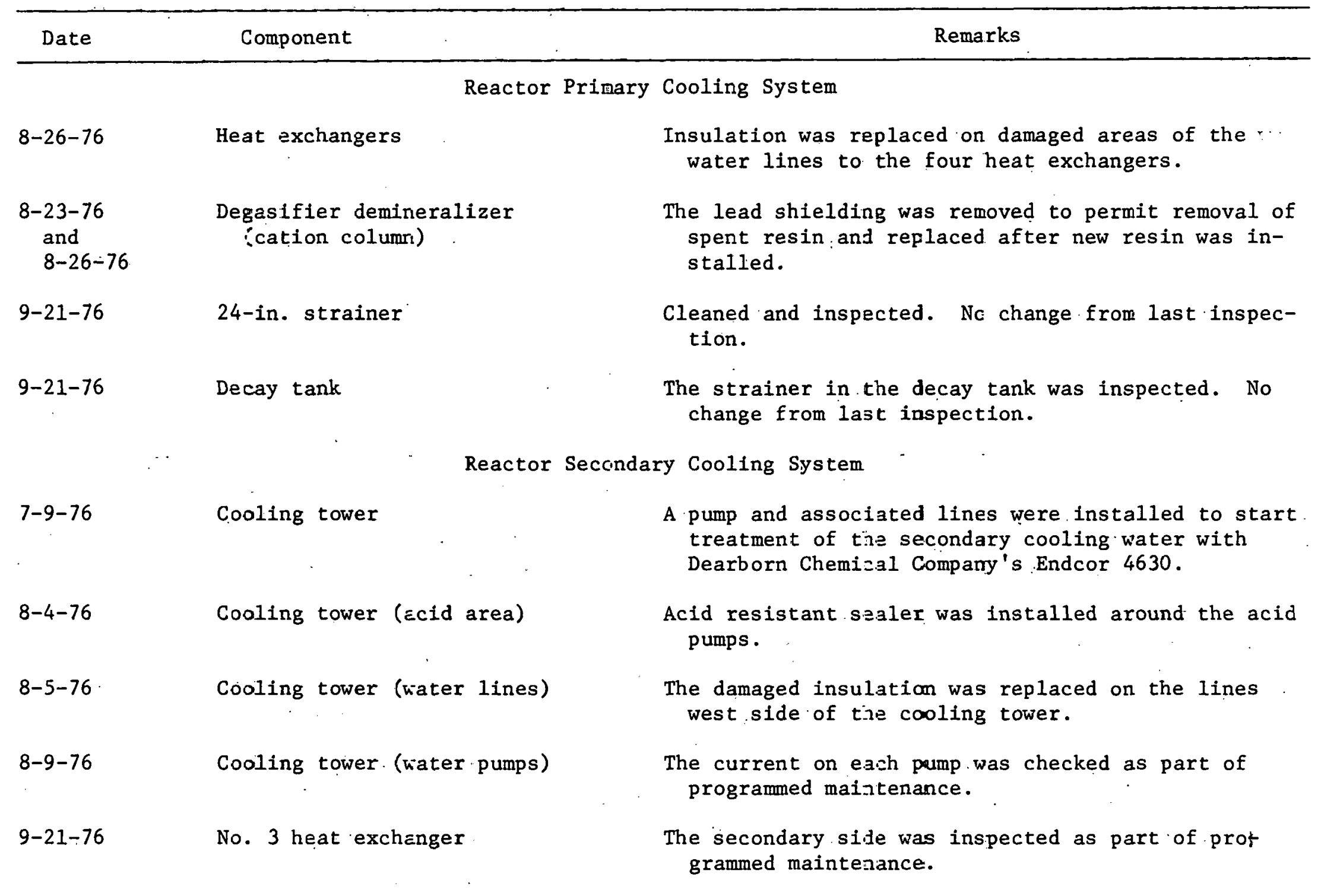


Table 9 (continued)

\begin{tabular}{lll}
\hline Date & Component & Remarks \\
\hline & Emergency Cooling System
\end{tabular}

8-6-76 Nos. 2 and 3 de units

Electrical Systems

$\begin{array}{ll}\text { 7-6-76 } & \text { Tele-alam system } \\ \text { 3-3-76 } & \begin{array}{l}\text { Transformer No. } 2 \\ \text { (Station 3-3) }\end{array} \\ \text { 3-4-76 } & \begin{array}{l}\text { Electrical breaker 4B } 41 \\ \text { (Station 4-4) }\end{array}\end{array}$

8-6-76 Sump pump No. 3

$8-3-76$

and

8-4-76

- 8-30-76

Diesel generatcr

Diese1 generator
New micro-sentry relays were installed in the No. 2 dc low amperage circuit.

A faulty annunciator alarm unit in the ORR control room was replaced.

A new transformer was installed at station 3-3 located on the east. side of Building 3001 .

The breaker located at station 4-4 was removed and sent to the electrical shop for a routine checkout. A meter base was installed at the station so a KWH meter could be installed at a later date.

A new electrical outlet for the pump was installed.

Emergency Electrical Systems
The routine end-of-cycle load test was performed. The governor was replaced because the: motor RPM cycled while test was.being performed.

A faulty oil heating thermostat was replaced. 
Table il. Status of Filters - Gaseous Waste System

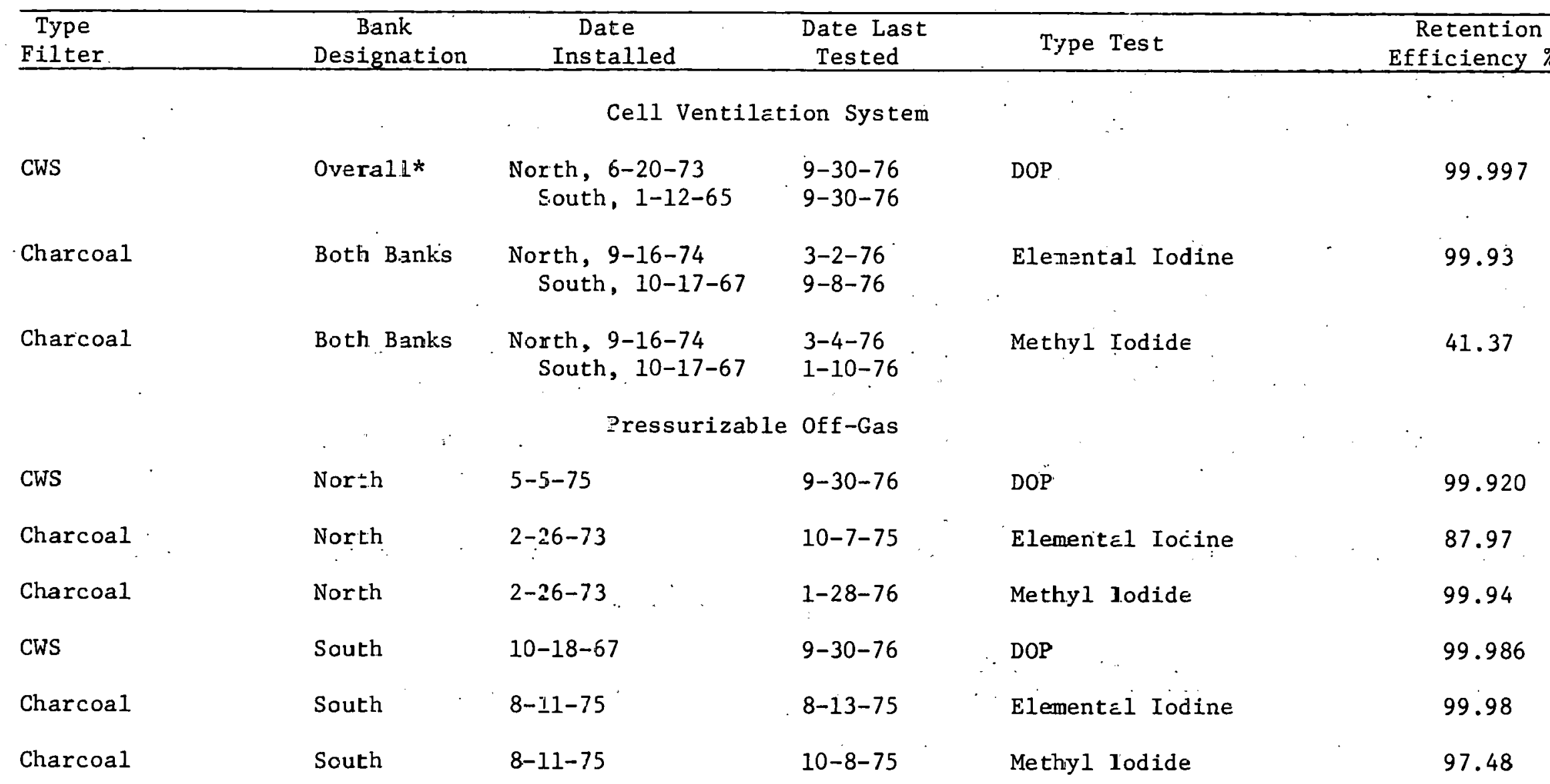

*The CWS filters in the cell ventilation system were checked in series. 
Table 11 (continued)

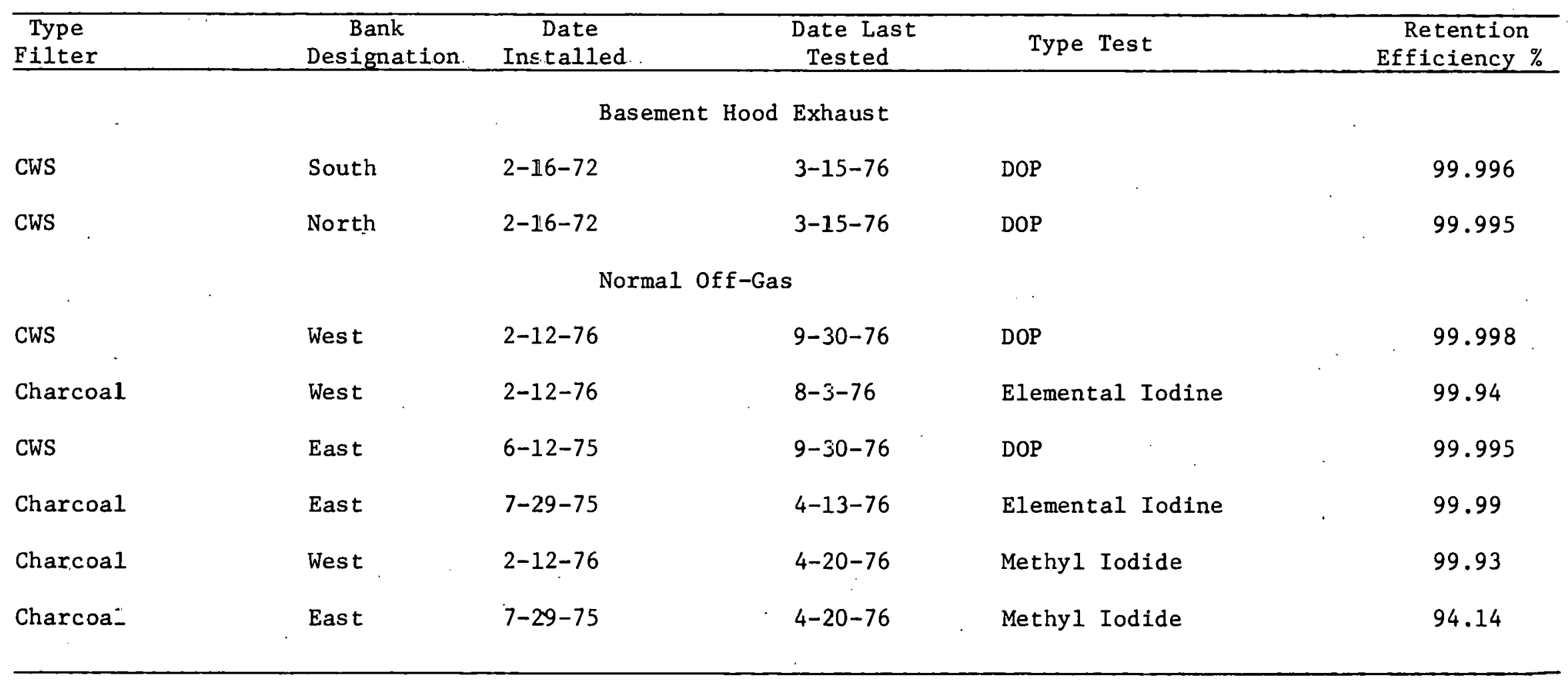


Table 12. Experimert Facility Usage

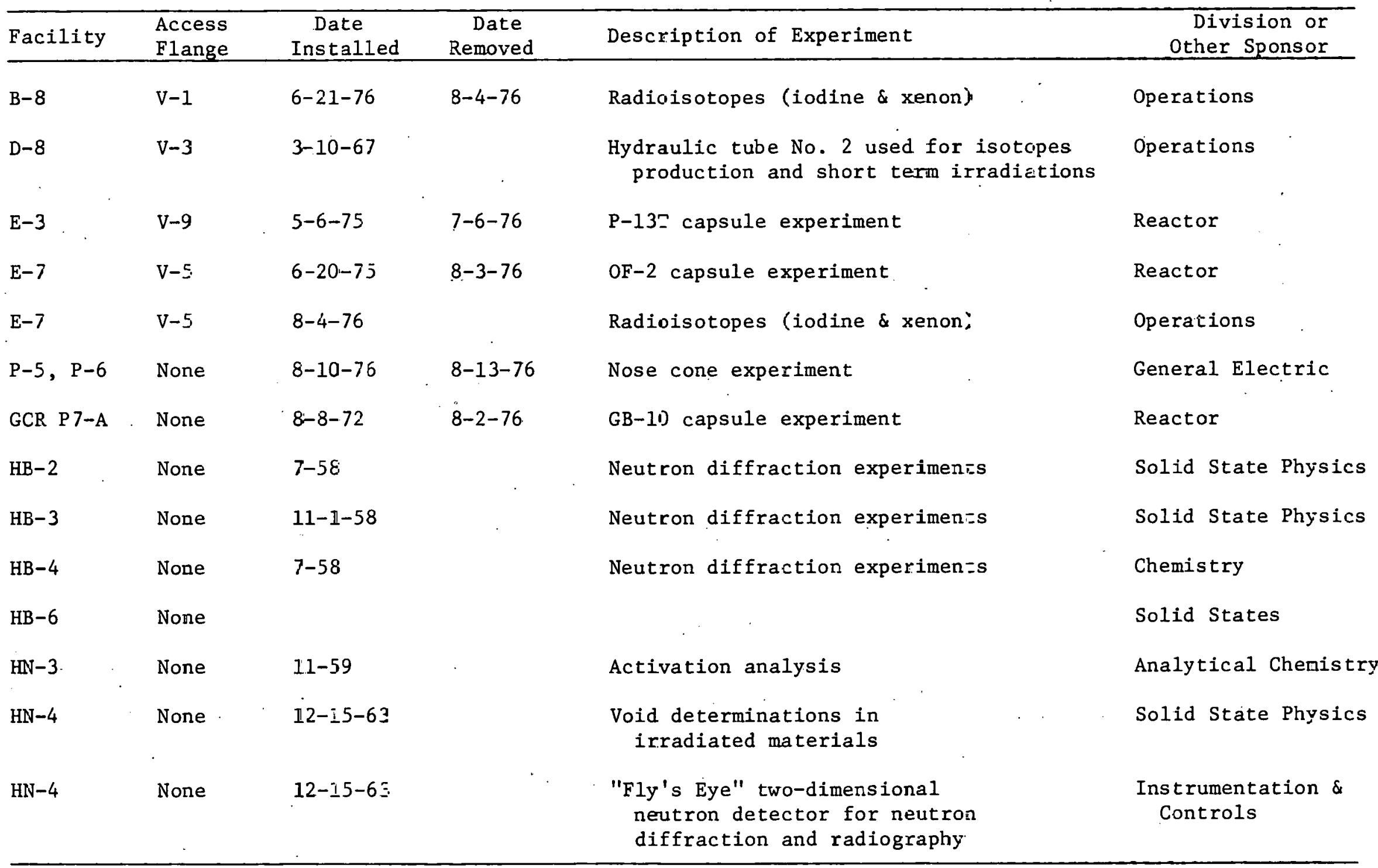


Table 13. Procedures, Additions, and/or Revisions

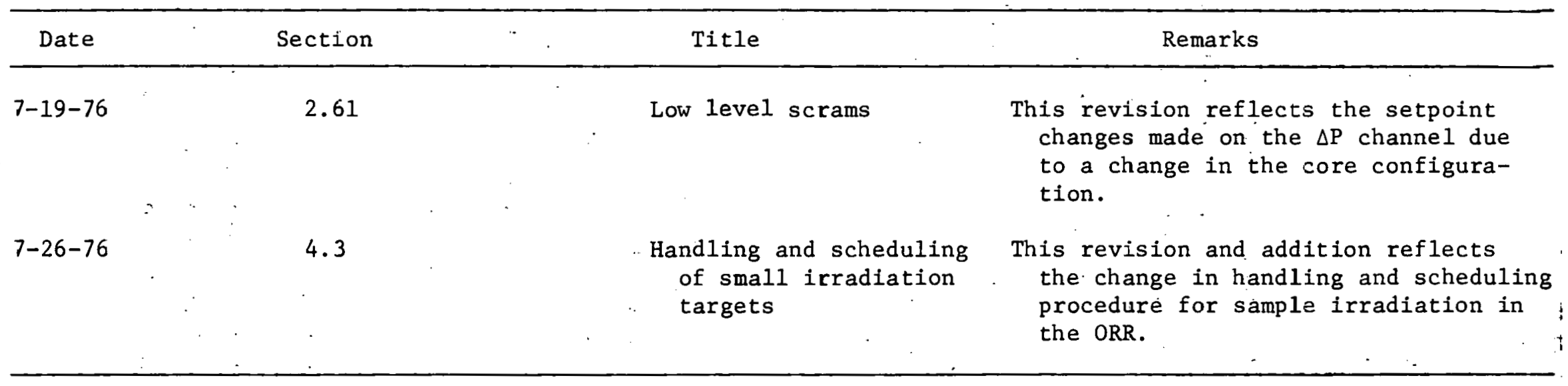




\section{IN-SERVICE INSPECTIONS}

\section{Decay Tank Inspection}

On 9-21-76, the water was drained down in the decay tank and an inspection of the decay tank strainer was made with satisfactory results.

2. Dearborn Chemical Division Consulting Service Report

On 7-1-76; switched from chromate to polyphosphate treatment of the ORR reactor secondary water. On 9-24-76 an inspection of the No. 3 heat exchanger secondary side Ind1cated " the heads and waler buxes to be very clean and free from any: heavy fouling or corrosion tubercules.

The tubes were very clean.with the exception of a light mineral deposition in the tubes on the outlet. This deposition appeared to extend approximately 2-3. ft. into the exchanger.

This deposition should pose no problems and should gradually clean up using the Endcor 4.630 and adhering to the proper pH and Ca levels recommended.

3. Visual Inspection of ORR Primary Coolant Heat Exchanger No. 3, Pit Area 3102

On 9-23-76, a visual. inspection of the secondary side of the subject heat exchanger revealed the tube-to-tube sheet welds to be in good.condition with the tubes proper being reasonably clean and free of obstructions (except in the thermocouple areas.) A pitting condition however existed on all visible head and shell surfaces with the maximum pit size measuring approximately $1 / 3$ inch in diameter by $3 / 64$ inch deep. (on. the return:closure head).

The general condition of the vessel is considered satisfactory for continued service.

4. Visual Inspection of ORR. 24-Inch Primary Coolant. Line. Strainer, Building 3085

On 9-23-76, a visual. inspection of the subject strainer revealed the four baskets to be secure, clean, and free of extraneous debris. The accessible surface of the housing and:closure head were also examined and found to be in satisfactory condition. 
ORNL/TM-5832

\section{INTERNAL DISTRIBUTION}

1. M. A. Baker

2. F. T. Binford

3. R. K. Branam

4. G. H. Burger

5. C. D. Cagle

6. W. R. Casto

7. G. H. Coleman

8. J. A. Conlin

9. J. A. Cox

10. F. L. Culler

11. S. J. Ditto

12. B. C. Duggins

13. W. A. Duggins

14. E. D. Gupton

15. T. P. Hamrick

16. V. O. Haynes

17. S. S. Hurt, III

18. J. D. Jenkins

19. L. P. Jernigan

20. E. B. Johnson

21. 0. L. Keller

22. E. M. King
23. Eugene Lamb

24. E. D. Lance

25. L. C. Lasher

26. D. L. Laughlin

27. R. V. McCord

28. F. H. Neill

29. T. B. Nixon

30. L. C. Oakes

31. Herman Postma

32. M. E. Ramsey

33. J. B. Ruble

34. T. M. Sims

35. M. J. Skinner

36. J. H. Swanks

37. J. R. Thomas

38. W. E. Thomas

39. K. R. Thoms

40. J. R. Weir, Jr.

41. K. W. West

42-44. Laboratory Records Department

45. Laboratory Records, ORNL R.C.

46-47. Central Research Library

48. Document Reference Section

EXTERNAL DISTRIBUTION

49. K. M. Akhtar, Pakistan

50-51. M. B. Biles, ERDA, Washington

52. Eric Blomberg, Sweden

53. K. J. Bobin, Belgium

54. ERDA-ORO: Safety and Environmental Control Division

55. C. Moller, Republic of South Africa

56. Director, Reactor Division, ERDA-ORO

57. Kiyoshi Inoue, Hitachi, Japan

58. M. Jacquemain, France

59. Masanori Kanbara, JAERI, Japan

60. W. Krul1, West German Federal Republic

61. Research and Technical Support Division, ERDA-ORO

62. 0. D. Oner, Turkey

63. G. Rada, Venerisela

64. R. J. Swanenburg de Veye, The Netherlands

65-91. Technical Information Center, Oak Ridge

92. R. Wakabayashi, NAIG, Japan

93. K. E. Wirtz, Germany

94. A. C. Woods, Australia 